\title{
Mycobacterium avium
}

National Cancer Institute

\section{Source}

National Cancer Institute. Mycobacterium avium. NCI Thesaurus. Code C86535.

A species of aerobic, Gram positive, rod shaped bacteria assigned to the phylum

Actinobacteria. This species is acid fast, catalase positive, niacin, peroxidase, nitrate reductase, and urease negative, and may be susceptible to pnitrobenzoate, ethambutol, pyrazinamide, rifampin, and streptomycin. M. avium is found in soil and dust particles and can cause infection when inhaled or ing ested, especially causing disseminated infection in immunocompromised patients. 